\title{
Influencia del uso de carbonato de calcio en la fertilización de Brachiaria decumbens y Brachiaria dictyoneura
}

\author{
Influence of the use of calcium carbonate in the fertilization of Brachiaria \\ decumbens and Brachiaria dictyoneura
}

\section{Influência do uso de carbonato de cálcio na fertilização de Brachiaria}

\section{decumbens e Brachiaria dictyoneura}

\author{
Morera Perilla Niyer Obed ${ }^{1}$, Riaño Peña Diego Mauricio y Silva Parra Amanda ${ }^{2}$ \\ 1I.A., Universidad de los Llanos y \\ 2..A., MSc., PhD., Docente Universidad de los Llanos \\ asilvap@unillanos.edu.co
}

Recibido 18 de Octubre de 2017, Aceptado 26 de Mayo 2018

\section{RESUMEN}

Este experimento se realizó en una región de clima cálido húmedo con precipitaciones entre 2700 y $3000 \mathrm{~mm} / \mathrm{año}$, con suelos ácidos y de baja fertilidad, cuyo objetivo fue evaluar la aplicación de carbonato de calcio $\left(\mathrm{CaCO}_{3}\right)$ y fertilizantes en dos tipos de gramíneas para establecer interacciones entre las propiedades químicas del suelo y producción de forraje. El cálculo de la cantidad de cal a aplicar (dosis total (DT)) se realizó con base en el porcentaje de aluminio tolerable, por lo cual los tratamientos fueron: $\mathrm{E} 1=0$ ton/ha (testigo), $\mathrm{E} 2=0.74$ ton/ha (1/3 de DT), E3 = 1.49 ton/ha (2/3 de DT) y E4 = 2.24 ton/ha ( $3 / 3$ de DT) de $\mathrm{CaCO}_{3}$ aplicado en todos los tratamientos después de la recuperación de la pastura a los 45 días. El diseño fue bloques completos al azar con arreglo factorial por tipo de gramínea (Brachiaria decumbens y Brachiaria dictyoneura), dosis de cal aplicada (4 enmiendas) y tres componentes de fertilización ( $N, P, y K)$, con tres repeticiones para un total de 72 unidades experimentales $(2 \times 4 \times 3 \times 3)$, cada una de $16 \mathrm{~m}^{2}$, factores que se relacionaron con materia orgánica $(\mathrm{MO})$, fósforo $(\mathrm{P}), \mathrm{pH}$, aluminio $(\mathrm{Al})$, calcio $(\mathrm{Ca})$, magnesio $(\mathrm{Mg})$, potasio $(\mathrm{K})$ y sodio $(\mathrm{Na})$. Las variables analizadas fueron: producción de forraje verde (FV) y materia seca (MS). El análisis estadístico se realizó mediante ANOVA con significancia del 95\%, y 
comparación de medias con el test de Tukey. El Al intercambiable en $B$. decumbens aumentó conforme incrementó la dosis de encalado, puesto que en E3 y E4 fue mayor a $2.2 \mathrm{cmol} / \mathrm{kg}$ de suelo; en tanto que con $B$. dictyoneura la dosis de cal $1 / 3$ del total lo disminuyó siendo menor a 1.98, aunque ambos valores seguían siendo altos en el suelo, indicando que las dosis aplicadas no lograron reducir el exceso de $\mathrm{Al}$, siendo diferentes entre sí $(\mathrm{P}<0.05)$. B. decumbens presentó mayor producción de FV y MS que $B$. dictyoneura $(P<0.05)$, la diferencia entre las producciones de biomasa fresca y seca en ambas especies de pastos, estuvo relacionada con las características propias de las pasturas y la tolerancia de $B$. decumbens a las condiciones de acidez y poca influencia de los factores limitantes como el exceso de $\mathrm{Al}$; bajo $\mathrm{pH}$ y contenido de $\mathrm{MO}$ y $\mathrm{P}$. La dosis de cal más alta con urea en E3 mejoró la eficiencia del $\mathrm{P}$ disponible en el suelo de las pasturas, igualmente generó una mayor producción de biomasa bajo condiciones de suelos ácidos. Potencializar los factores de producción de $B$. dictyoneura en la zona sería importante para mejorar su producción, puesto que con esta especie se incrementa la $\mathrm{MO}$, el $\mathrm{P}$ disponible, y se reduce el $\mathrm{Al}$ intercambiable y el $\mathrm{pH}$; en comparación con $B$. decumbens, cuando se fertiliza con $\mathrm{N}$ se mejoran los contenidos de $\mathrm{MO}$, por otra parte, con todas las dosis de encalado evaluadas se redujo el Al intercambiable del suelo.

Palabras clave: Gramínea, suelos ácidos, producción de biomasa, pasturas, ganadería.

\section{ABSTRACT}

This experiment was carried out in a humid warm climate region with rainfall between 2700 and $3000 \mathrm{~mm} /$ year, with acid and low fertility soils, whose objective was to evaluate the application of calcium carbonate $\left(\mathrm{CaCO}_{3}\right)$ and fertilizers in two types of grasses to establish interactions between soil chemical properties and forage production. The calculation of the amount of lime to be applied (total dose (TD)) was made based on the percentage of tolerable aluminum, for which the treatments were: $\mathrm{E} 1=0$ ton/ha (control), $\mathrm{E} 2=0.74$ ton/ha $(1 / 3$ of TD), $\mathrm{E} 3=1.49$ ton/ha $\left(2 / 3\right.$ of TD) and $\mathrm{E} 4=2.24$ ton/ha $\left(3 / 3\right.$ of TD) of $\mathrm{CaCO}_{3}$ applied in all 
treatments after the recovery of the pasture at 45 days. The design was randomized complete blocks with factorial arrangement by type of grass (Brachiaria decumbens and Brachiaria dictyoneura), applied dose of lime (4 amendments) and three components of fertilization ( $N, P$, and $K)$, with three repetitions for a total of 72 experimental units $(2 \times 4 \times 3 \times 3)$, each one of $16 \mathrm{~m}^{2}$, factors that were related to organic matter $(\mathrm{OM})$, phosphorus $(\mathrm{P}), \mathrm{pH}$, aluminum $(\mathrm{Al})$, calcium $(\mathrm{Ca})$, magnesium $(\mathrm{Mg})$, potassium $(\mathrm{K})$ and sodium $(\mathrm{Na})$. The variables analyzed were: green forage production (FV) and dry matter (DM). The statistical analysis was performed using ANOVA with $95 \%$ significance, and comparison of means with the Tukey test. The Al exchangeable in $B$. decumbens increased as the liming dose was increased, because in E3 and E4 it was greater than 2.2 $\mathrm{cmol}_{\mathrm{c}} \mathrm{kg}$ of soil; while with $B$. dictyoneura the dose of lime $1 / 3$ of the total decreased it being less than 1.98, although both values remained high in the soil, indicating that the doses applied did not manage to reduce the excess of $\mathrm{Al}$, being different from each other $(\mathrm{P}<0.05)$. $B$. decumbens showed higher production of $F V$ and MS than $B$. dictyoneura $(P<0.05)$, the difference between the production of fresh and dry biomass in both pasture species, was related with the own characteristics of the pastures and the tolerance of $B$. decumbens to the conditions of acidity and little influence of the limiting factors such as the excess of $\mathrm{Al}$, low $\mathrm{pH}$ and content of $\mathrm{OM}$ and $\mathrm{P}$. The highest dose of lime with urea in E3 improved the efficiency of the $\mathrm{P}$ available in the soil of the pastures, it also generated a higher biomass production under acid soil conditions. Potentializing the production factors of $B$. dictyoneura in the area would be important to improve its production, because with this species is increased the $\mathrm{MO}$, the $\mathrm{P}$ available, and the exchangeable $\mathrm{Al}$ and $\mathrm{pH}$ are reduced; in comparison with $B$. decumbens, when is fertilized with $\mathrm{N}$, the OM contents are improved; on the other hand, with all the liming doses evaluated, the exchangeable Al of the soil was reduced.

Keywords: Grass, acid soils, biomass production, pastures, livestock. 


\section{RESUMO}

Este experimento foi realizado em uma região de clima quente úmido, com precipitações entre 2700 e 3000 mm/ano, com solos ácidos e de baixa fertilidade, cujo objetivo foi avaliar a aplicação de carbonato de cálcio $\left(\mathrm{CaCO}_{3}\right)$ e fertilizantes em dois tipos de gramíneas para estabelecer interações entre propriedades químicas do solo e produção de forragem. O cálculo da quantidade de cal a aplicar (dose total (DT)) foi feito com base na percentagem de alumínio tolerável, palo qual os tratamentos foram: $\mathrm{E} 1=0$ ton/ha (controle), $\mathrm{E} 2=0.74$ ton/ha $(1 / 3$ do DT), $\mathrm{E} 3=1.49$ ton/ha (2/3 do DT) e E4 = 2.24 ton/ha (3/3 do DT) de $\mathrm{CaCO}_{3}$ aplicado em todos os tratamentos após a recuperação do pasto aos 45 dias. $O$ delineamento experimental foi blocos completos casualizados com arranjo fatorial por tipo de grama (Brachiaria decumbens e Brachiaria dictyoneura), dose aplicada de cal (4 emendas) e três componentes de fertilização ( $N$, P e K), com três repetições para um total de 72 unidades experimentais $(2 \times 4 \times 3 \times 3)$, cada uma com $16 \mathrm{~m}^{2}$, fatores que foram relacionados com a matéria orgânica $(\mathrm{MO})$, fósforo $(P)$, $\mathrm{pH}$, alumínio $(\mathrm{Al})$, cálcio $(\mathrm{Ca})$, magnésio $(\mathrm{Mg})$, potássio $(\mathrm{K})$ e sódio $(\mathrm{Na})$. As variáveis analisadas foram: produção de forragem verde (FV) e matéria seca (MS). A análise estatística foi realizada por meio da ANOVA, com significância de 95\%, e comparação de médias com o teste de Tukey. O Al trocável em $B$. decumbens aumentou com o aumento da dose de cal aplicada, já que em E3 e E4 foi maior a $2.2 \mathrm{cmol} / \mathrm{kg}$ de solo; enquanto que com $B$. dictyoneura a dose de cal 1/3 do total diminuiu sendo menor que 1.98 , embora ambos os valores permanecessem elevados no solo, indicando que as doses aplicadas não conseguiram reduzir o excesso de $A \mathrm{l}$, sendo diferentes um do outro $(P<0.05)$. B. decumbens apresentou maior produção de FV e MS que $B$. dictyoneura $(P<0.05)$, a diferença entre a produção de biomassa fresca e seca em ambas as espécies de pastagem, estava relacionada com características próprias das pastagens e a tolerância de $B$. decumbens a condições de acidez e pouca influência de fatores limitantes, como o excesso de $\mathrm{Al}$, baixo $\mathrm{pH}$ e conteúdo de $\mathrm{MO}$ e $\mathrm{P}$. A maior dose de calcário com uréia em E3 melhorou a eficiência do $\mathrm{P}$ disponível no solo das pastagens, também gerou uma maior produção de biomassa em condições de solo ácido. 
Potencializar os fatores de produção de $B$. dictyoneura na área seria importante para melhorar sua produção, já que com esta espécie aumenta o $\mathrm{MO}, \mathrm{P}$ disponível, e o $\mathrm{Al}$ e pH trocáveis são reduzidos; em comparação com B. decumbens, quando é fertilizado com N, o conteúdo de MO é melhorado; por outro lado, com todas as doses de cal avaliadas, o Al trocável do solo foi reduzido.

Palavras-chave: Grama, solos ácidos, produção de biomassa, pastagens, gado.

\section{INTRODUCCIÓN}

Las pasturas que son la base de la alimentación de los bovinos, se han introducido posterior a la evaluación y selección por su adaptación en suelos con alta saturación de aluminio (Al); se ha demostrado que Brachiaria decumbens, tiene capacidad de acumular altas concentraciones de este mineral en las raíces lo que favorece la síntesis de malato, aunque se disminuye su rendimiento (Hartwig et al., 2007). Es importante señalar que el Al puede afectar a la planta, interfiriendo en su absorción de nutrientes esenciales como calcio $(\mathrm{Ca})$, magnesio $(\mathrm{Mg})$ y potasio $(\mathrm{K})$, además en niveles altos es tóxico para las raíces (Sanchez y Salinas, 2008). El desarrollo de genotipos de pastos tolerantes al aluminio ha contribuido al incremento de la producción de biomasa en los suelos ácidos de los trópicos.

El $\mathrm{Al}^{+3}$ en la solución del suelo es el principal responsable de la disminución en el rendimiento de las plantas, debido a que inhibe el desarrollo de las raíces (Zapata, 2004). Altos niveles de saturación de $\mathrm{Al}^{+3}$ también reducen su crecimiento, absorción de agua y nutrimentos, inhibiendo así su elongación y penetración en el suelo, dificultando que las raíces lleguen al subsuelo, y por lo tanto se obstaculiza la translocación de nutrimentos a la parte aérea, los cuales se manifiestan principalmente como deficiencias de nitrógeno $(\mathrm{N})$, fósforo $(\mathrm{P})$ y potasio $(\mathrm{K})$ ( $\mathrm{Li}$ y Johnson, 2016).

Los fertilizantes son insumos de elevado valor que representan un importante porcentaje en los costos de establecimiento y mantenimiento de las praderas, porque la corrección de la acidez de los suelos con el uso de carbonato de calcio $\left(\mathrm{CaCO}_{3}\right)$ puede ayudar a mejorar su eficiencia y disponibilidad de los nutrientes en 
el suelo para alcanzar mayores rendimientos. El uso de esta sustancia correctiva como complemento de la fertilización puede ser también una práctica que da muy buenos resultados en praderas de Brachiaria decumbens ya establecidas en suelos ácidos (Anchão et al., 2003; Rincón et al., 2010), pero esta práctica es poco frecuente entre los ganaderos por lo que no hay un resultado claro y visible de su efecto cuando se combina con fertilizantes. Castro y Munevar, (2013) reportan que el $\mathrm{CaCO}_{3}$ es el principal componente de la piedra caliza, y se utiliza como enmienda para neutralizar la acidez del suelo y suministrar calcio (Ca) para la nutrición de las plantas. Este componente reduce el Al soluble por dos reacciones:

$$
\begin{gathered}
\mathrm{CaCO}_{3}+\mathrm{H}_{2} \mathrm{O} \rightarrow \mathrm{Ca}^{2+}+2 \mathrm{OH}^{-}+\mathrm{CO}_{2} \\
\mathrm{Al}^{3+}+[\text { soluble }]+3 \mathrm{OH}^{-} \rightarrow \mathrm{Al}(\mathrm{OH})_{3}[\text { insoluble }]_{20}
\end{gathered}
$$

Jiménez et al., (2010) afirman que la pastura se utiliza como base de alimentación de los bovinos y que el retorno de nutrientes al suelo se realiza de manera natural a través de hojarasca producida por el pastizal y por las excretas de animales; sin embargo, altas intensidades de pastoreo, reducen la acumulación de hojarasca y el reciclaje de nutrientes se produce solamente a través de las excretas animales, lo cual no es suficiente (Rodríguez et al., 2001), por lo tanto se requiere aportar al suelo los nutrientes necesarios para mantener de manera constante la producción de biomasa, puesto que la fertilización afecta la composición química del pasto, haciendo más disponible el elemento que se aplica.

La acelerada acidificación de muchos suelos bajo el esquema del mejoramiento de las pasturas, que son fertilizadas con nitrógeno en varias partes del mundo, elemento que en gran parte, se lixivia en forma de nitrato y no puede ser utilizado por la planta, participa en una amplia proporción en la acidificación de los suelos, estimándose que entre 14 a $80 \mathrm{~kg}$ de nitrógeno en forma de nitrato puede ser lixiviado por hectárea al año, aun cuando los requerimientos pueden alcanzar hasta $400 \mathrm{~kg}$ de N/ha en pasturas de alta producción, aunque tienen elevadas pérdidas (hasta 200 kg N/ha) (Arias et al., 2004; Civeira y Rodríguez, 2011) 
El fósforo, es un mineral, que se afecta en su disponibilidad por la acidez del suelo, que desde el punto de vista agronómico y de producción vegetal, cumple funciones como: estimulación del crecimiento y expansión foliar, promoción del crecimiento de las raíces y mejoramiento de la calidad nutricional de la pastura, por lo tanto, el fósforo es un macronutriente esencial para el crecimiento y desarrollo de las praderas (Rincón et al., 2012). También se ha demostrado la escasa respuesta de la pradera natural establecida en suelos ácidos a las aplicaciones de potasio desde el primer que se aumenta a través de los diferentes cortes (Castro y Gómez, 2010). Diferentes estudios han demostrado que los suelos ácidos limitan la disponibilidad y absorción de $\mathrm{N}, \mathrm{K}^{+}, \mathrm{Ca}^{2+}, \mathrm{Mg}^{2+}$ y $\mathrm{P}$ (Quintero et al., 2013), con respecto a esto, existen reportes sobre el uso benéfico de materiales como $\mathrm{CaCO}_{3}$ para elevar el $\mathrm{pH}$ de los suelos ácidos (Auclerc et al., 2012).

\section{METODOLOGÍA}

Este experimento se realizó en una zona del Piedemonte Llanero ubicado en la región de la Orinoquia de Colombia, con alturas que van desde los 200 a 700 msnm, la altitud en la zona es de $430 \mathrm{msnm}$ (CORPOICA, 2007). De acuerdo con el sistema de clasificación de Koeppen, el clima es tropical húmedo y seco (clima de sabana), con épocas bien definidas de lluvia y de sequía, con promedios de precipitación de 2700 a $3000 \mathrm{~mm} / a n ̃ o$, temperatura de $28^{\circ} \mathrm{C}$ y humedad relativa del $85 \%$.

Los tratamientos se establecieron de acuerdo con el factor tipo de pastura: B1= Brachiaria decumbens y $\mathrm{B} 2=$ Brachiaria dictyoneura; tres componentes de fertilización: $\mathrm{N}, \mathrm{P}$ y $\mathrm{K}$, siendo $\mathrm{N}=$ urea $(46 \%$ de $\mathrm{N}), \mathrm{P}=\left(46 \% \mathrm{P}_{2} 0_{5}\right), \mathrm{K}=\mathrm{KCl}(65 \%$ $\mathrm{K}_{2} \mathrm{O}$ ), y cuatro dosis $\mathrm{CaCO}_{3}$ (cal), las cuales se determinaron mediante la metodología de Cochrane et al., (1980) teniendo en cuenta que el Al intercambiable fue de $1.90 \mathrm{cmol} / \mathrm{kg}$, Ca $0.55 \mathrm{cmol} / \mathrm{kg}, \mathrm{Mg} 0.05 \mathrm{cmol} / \mathrm{kg}, \mathrm{K} 0.09$ $\mathrm{cmol}_{\mathrm{c}} / \mathrm{kg}$, la sumatoria da como resultado la capacidad de intercambio catiónico efectiva (CICE), $2.61 \mathrm{cmol} / \mathrm{kg}$, porcentaje de aluminio tolerable por el cultivo $25 \%$, y se realizó el encalado así: E1= cero ton/ha (testigo); E2=0.74 ton/ha (1/3 de la 
dosis total (DT)); E3= 1.49 ton/ha (2/3 de la DT) y E4=2.24 ton/ha (3/3 de la DT de $\mathrm{CaCO}_{3}$. Estas dosis se definieron con base en un análisis inicial de suelo realizado en laboratorio, donde 2.74 ton/ha es la dosis de cal (encalado) recomendada (Salinas, 1989), aplicando la siguiente fórmula:

Toneladas de $\mathrm{CACO}_{3}($ ton $/ h a)=1.8 X(A l-R A S(A l+C a+M g)) / 100 g$ de suelo

Donde RAS es el máximo porcentaje de aluminio intercambiable tolerado por el cultivo. Todos los tratamientos se evaluaron en un solo corte después de la recuperación de la pastura a los 45 días. El diseño experimental fue de bloques completos al azar con arreglo factorial, donde el primer factor correspondió a los tipos de pasturas, el segundo a las cuatro dosis de cal y el tercero a los tres componentes de fertilización: $N, P, y \mathrm{~K}$; factores que se relacionaron con el contenido de materia orgánica (MO), $\mathrm{P}, \mathrm{pH}, \mathrm{Al}, \mathrm{Ca}, \mathrm{Mg}, \mathrm{K}$ y $\mathrm{Mg}$. El total de unidades experimentales fue el resultado de las interacciones entre tres estos factores: dos pastos, cuatro dosis de $\mathrm{CaCO}_{3}$, tres componentes de fertilización y tres repeticiones $(2 \times 4 \times 3 \times 3)$, para un total de 72 unidades experimentales, cada una de $16 \mathrm{~m}^{2}$, que corresponde a un total de $1.152 \mathrm{~m}^{2}$ de área experimental, siendo el área útil de cada parcela $12 \mathrm{~m}^{2}$, evitando así el efecto de bordes.

Las variables analizadas fueron producción de forraje verde (FV) y materia seca (MS), para lo cual a los 45 días post-aplicación de tratamientos se realizaron aforos en cada unidad experimental con marcos cuadrados de $40 \mathrm{~cm}$, y posteriormente se extrapolaron los datos a ton/ha. Las submuestras de FV se secaron a $65^{\circ} \mathrm{C}$ durante 72 horas en estufa de ventilación forzada, para estimar el porcentaje de materia seca parcial por diferencia de peso antes y después del secado (Posada et al., 2007). El análisis estadístico de los datos se realizó mediante ANOVA con un nivel de significancia del 95\%, y en la comparación de medias se aplicó el test de Tukey $(\mathrm{P}<0.05)$, utilizando el paquete estadístico INFOSTAT versión 2017 (Di Renzo et al., 2017). 


\section{RESULTADOS Y DISCUSIÓN}

El análisis de suelo evidencia bajos niveles de $\mathrm{MO}$, indicando que la actividad de los organismos del suelo (biota), incluyendo los microorganismos, es reducida, afectando la descomposición de los residuos que son posteriormente forman la $\mathrm{MO}$, en consecuencia nutrientes como nitrógeno, fósforo y azufre no son liberados dentro del suelo en formas que pueden ser usadas por las plantas, afectando así la disponibilidad de nutrientes, por lo cual el contenido de minerales es bajo, haciendo que estos suelos tengan una baja fertilidad. Este suelo tiene una reacción ácida, con pH inferior a 5.5, siendo la acidificación una de las causas más importantes de degradación de los suelos, porque es un proceso químico en el que aumentan los hidrogeniones $\left(\mathrm{H}^{+}\right)$, al mismo tiempo que se disminuye la concentración de cationes básicos como calcio $\left(\mathrm{Ca}^{2+}\right)$, magnesio $\left(\mathrm{Mg}^{2+}\right)$, sodio $\left(\mathrm{Na}^{+}\right)$y potasio $\left(\mathrm{K}^{+}\right)$con un alto contenido de aluminio que se solubiliza y se convierte en su catión trivalente $\mathrm{Al}^{3+}$, que en altas cantidades es tóxico para la planta. La acidez del suelo hace que las raíces estén siempre expuestas al aluminio lo cual afecta su producción.

Tabla 1. Análisis de suelo de las parcelas establecidas con Brachiaria dictyoneura y B. decumbens

\begin{tabular}{cccc}
\hline & Parámetros & \\
\hline $\mathrm{MO} \%$ & 1.7 & $\mathrm{P}(\mathrm{pmm})$ & 2.9 \\
$\mathrm{pH}$ & 4.6 & $\mathrm{Cu}(\mathrm{ppm})$ & 1.8 \\
$\mathrm{Al}(\mathrm{mq} / 100 \mathrm{gr}$ de suelo $)$ & 1.90 & $\mathrm{Fe}(\mathrm{ppm})$ & 163.37 \\
$\mathrm{Ca}(\mathrm{mq} / 100 \mathrm{gr}$ de suelo $)$ & 0.55 & $\mathrm{Mn}(\mathrm{ppm})$ & 31.25 \\
$\mathrm{Mg}(\mathrm{mq} / 100 \mathrm{gr}$ de suelo $)$ & 0.05 & $\mathrm{Zn}(\mathrm{ppm})$ & 0.55 \\
$\mathrm{~K}(\mathrm{mq} / 100 \mathrm{gr}$ de suelo $)$ & 0.09 & $\mathrm{~B}(\mathrm{ppm})$ & 0.18 \\
$\mathrm{Na}(\mathrm{mq} / 100 \mathrm{gr}$ de suelo $)$ & 0.02 & $\mathrm{~S}(\mathrm{ppm})$ & 4.78 \\
\hline
\end{tabular}

$\mathrm{MO}=$ Materia orgánica. Tipo de suelo: Franco arcilloso

Metodología: MO: Walkley black. S: Fosfato monobásico de Calcio. Cationes: $\mathrm{ACNH}_{4}, 1 \mathrm{~N}, \mathrm{pH} 7,0$.

Elementos menores DIPA. Al: HC 1N. B: en frio HCL, 0,03M. P: Bray II. PH: 1:1 suelo: agua 
El Al intercambiable en $B$. decumbens aumentó con las dosis de encalado más altas, E3 y E4 (mayor de 2,2 cmolc/kg de suelo), en tanto que con $B$. dictyoneura la dosis de cal $1 / 3$ del total lo disminuyó siendo menor a 1.98, ambos valores siguen siendo altos en el suelo (Figura 1), lo que indica que las dosis aplicadas no lograron reducir el $\mathrm{Al}$, aunque la respuesta si fue diferente en función de la cantidad aplicada $(\mathrm{P}<0.05)$.

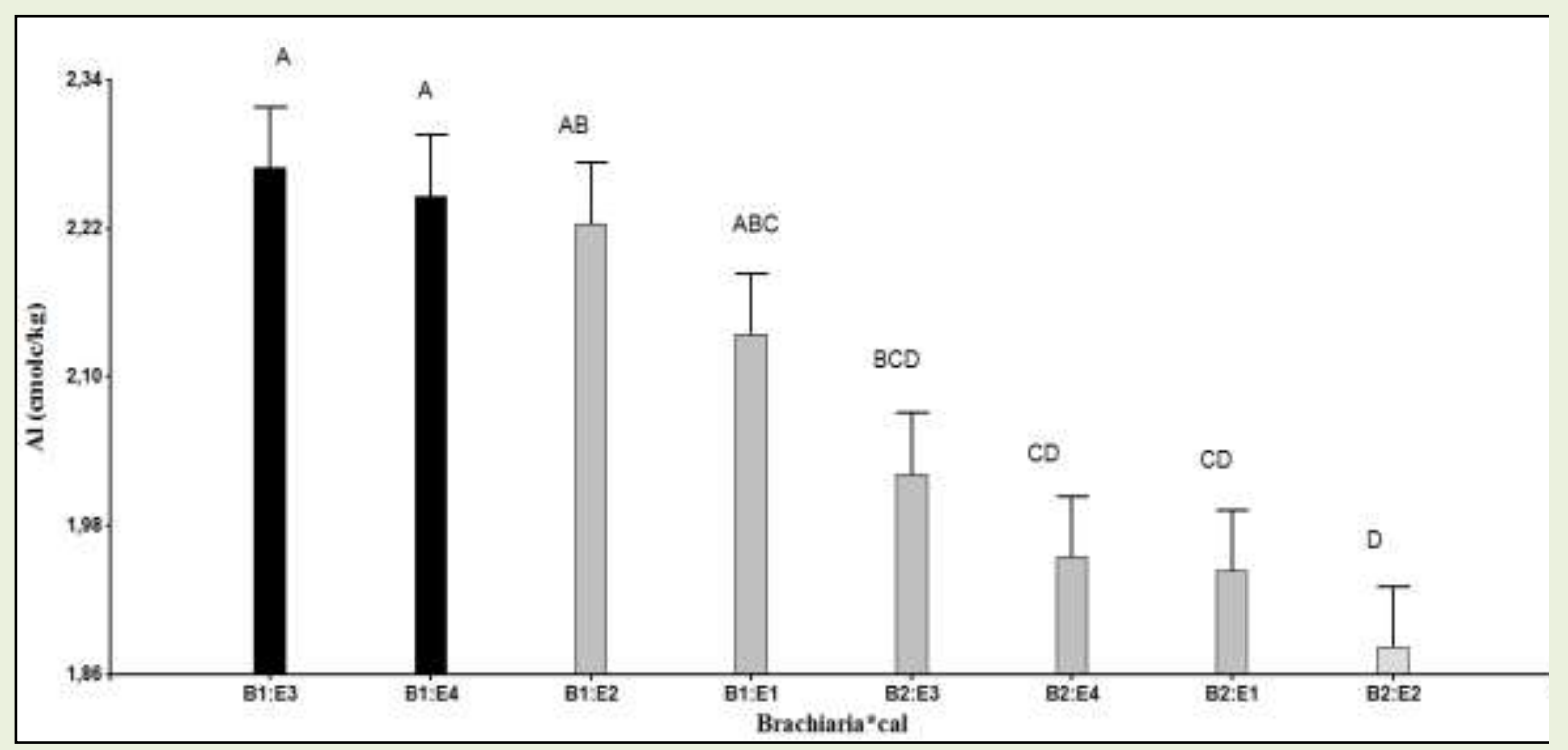

$\mathrm{E} 1=$ sin cal. $\mathrm{E} 2=0.74, \mathrm{E} 3=1.49$ y $\mathrm{E} 4=2.24$ toneladas de carbonato de calcio $\left(\mathrm{CaCO}_{3}\right)$ por hectárea. $\mathrm{B} 1=$ Brachiaria decumbens, $\mathrm{B} 2=$ Brachiaria dictyoneura.

Letras distintas en la figura indican diferencias estadísticas significativas $(P<0.05)$.

Figura 1. Efecto del factor tipo de gramínea la dosis de cal sobre el Al intercambiable

Las comparaciones entre $B$. decumbens y $B$. dictyoneura con relación a las propiedades químicas del suelo presentaron efectos $(\mathrm{P}<0.05)$ en $\mathrm{MO}, \mathrm{P}, \mathrm{pH}, \mathrm{Al}$ y $\mathrm{Mg}$. En $B$. dictyneura los promedios de $\mathrm{MO}, \mathrm{P}$ y $\mathrm{pH}$ fueron $1.58 \%, 2.13 \mathrm{ppm}$ y 5.12, mayores que en $B$. decumbens $1.11 \%, 0.70$ ppm y 5.0 respectivamente; (Figura 2), en ambos casos los contenidos están clasificadas según el IGAC, (2003) como bajos para la zona. En $B$. decumbens se encontraron mayores contenidos de $\mathrm{Al}$ intercambiable (2.22 $\mathrm{cmol} / \mathrm{kg}$ de suelo) en comparación con la otra gramínea $(1.95 \mathrm{cmol} / \mathrm{kg}$ de suelo) $(\mathrm{P}<0.05)$ (Figura 2$)$. 

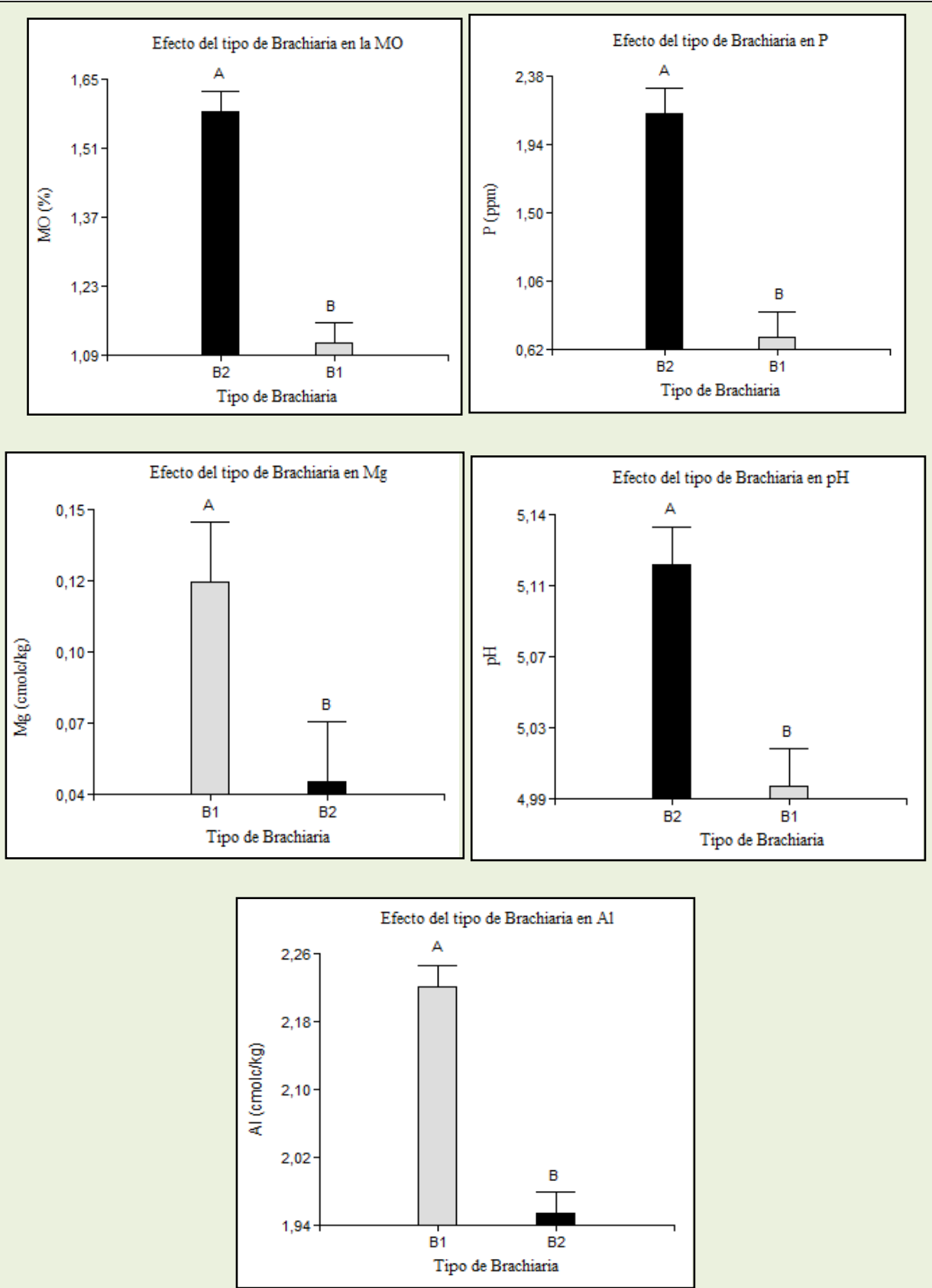

$\mathrm{B} 1=$ Brachiaria decumbens, $\mathrm{B} 2=$ Brachiaria dictyoneura .

Letras distintas en la figura indican diferencias estadísticas significativas $(P<0.05)$.

Figura 2. Interacciones de las propiedades químicas en los suelos donde se sembraron los dos tipos de gramínea

Según el IGAC, (2003) estos valores tipifican a los suelos con alta acidez intercambiable puesto que son mayores a $1 \mathrm{cmol} / \mathrm{kg}$ de suelo; el $\mathrm{Mg}$ fue superior a $0.10 \mathrm{cmolc} / \mathrm{kg}$ de suelo en $B$. decumbens $(0.12)(\mathrm{P}<0.05)$ y menor a 0.07 en $B$. 
dictyneura (0.05), considerados como bajos $(P<0.05)$, siendo menores 1.5 $\mathrm{cmol}_{\mathrm{c}} / \mathrm{kg}$ de suelo, que es lo estipulado para que un suelo sea adecuado en el contenido de este mineral. Por otro lado, la dosis de cal más alta junto con nitrógeno mejoró la eficiencia del fósforo en el suelo con valores que oscilaron entre 1.77 a 2.27 ppm, a diferencia del caso en que el que se utilizó potasio, donde dicho contenido fue menor a $1.26 \mathrm{ppm}$, siendo diferentes a las otras interacciones evaluadas $(P<0.05)$ (Figura 3$)$.

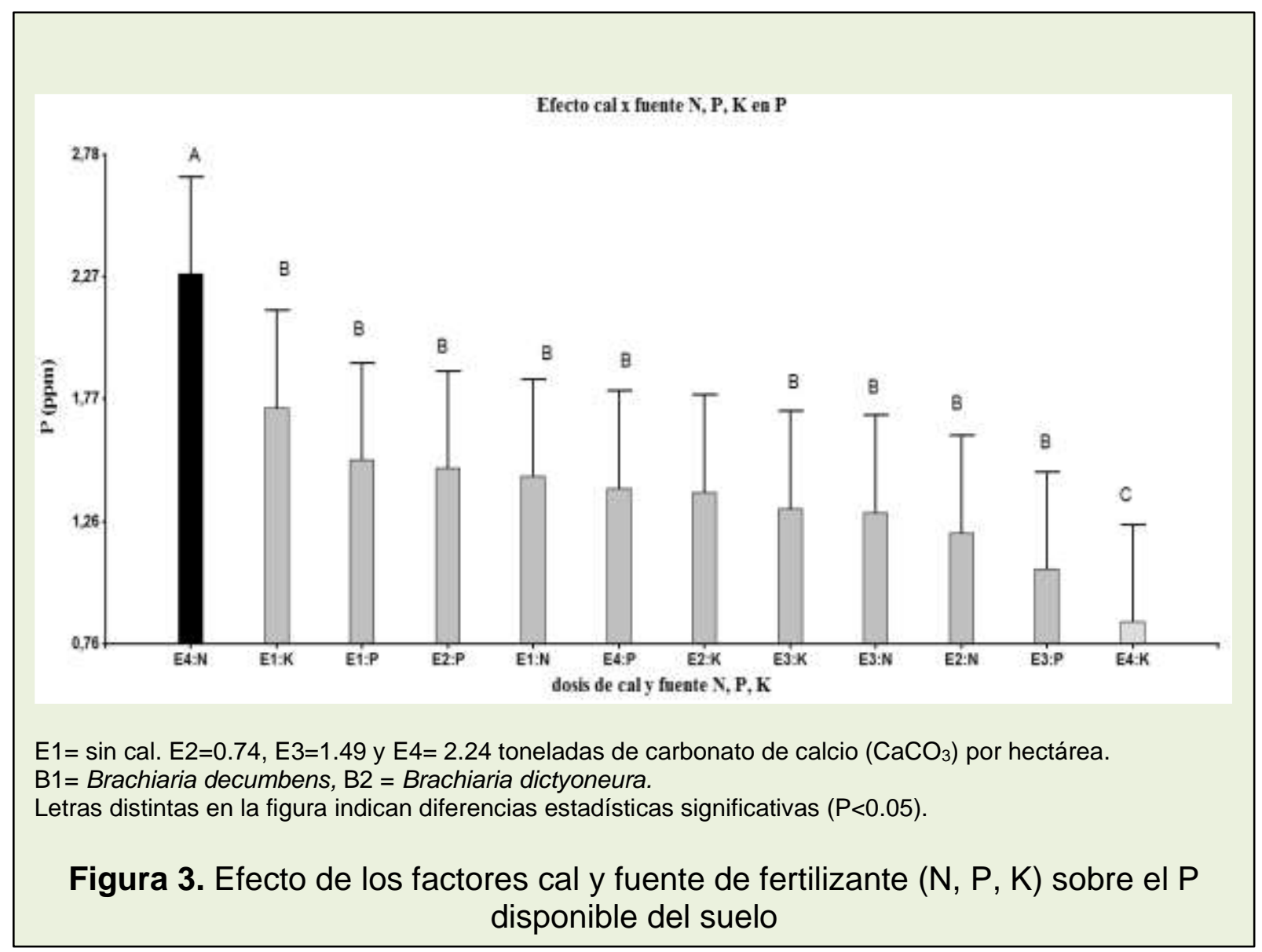

Se observó que el efecto en los contenidos de Al estuvo influenciado por el tipo de gramínea y no por las dosis crecientes de cal, puesto que en $B$. decumbens este mineral aumentó con las dosis más altas de cal en E3 y E4, con valores medios que oscilaron entre 2.22 y $2.34 \mathrm{cmol} / \mathrm{kg}$ de suelo respectivamente $(P<0.05)$, siendo diferente a $B$. dictyneura que presentó un menor contenido de Al intercambiable con la dosis de cal más baja E2, entre 1.86 y $1.98 \mathrm{cmol} / \mathrm{kg}$ de 
suelo $(P<0.05)$. Por lo tanto no hubo una relación directa entre los aumentos en las dosis de cal con la disminución del Al intercambiable del suelo (Figura 4).

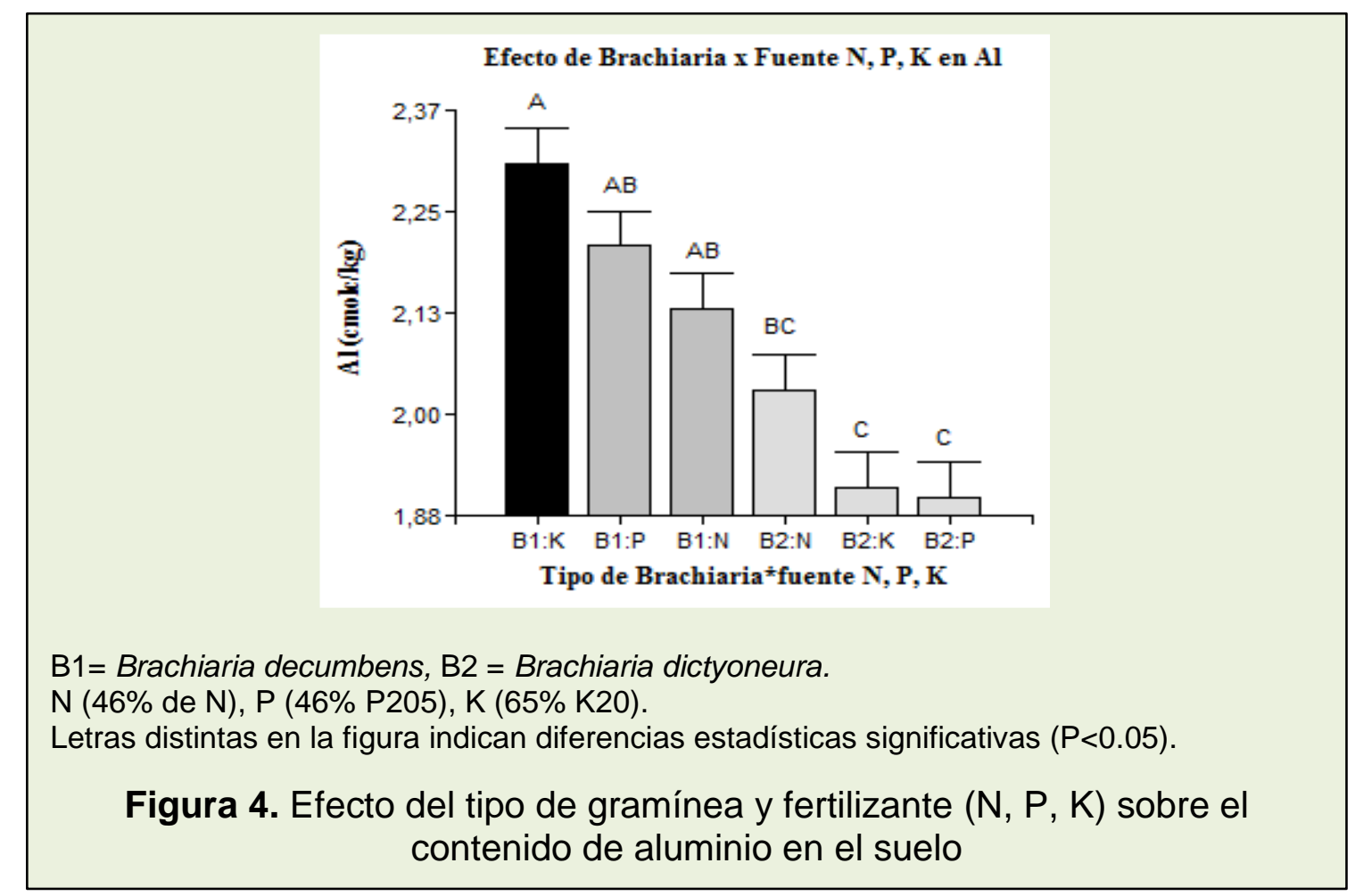

La producción de forraje verde (FV) (Figura 5) respondió positivamente a la aplicación de cal (E3) con nitrógeno (mayor a 8.38 toneladas de FV por hectárea), pero fue menor a E4 con fósforo y potasio, que presentaron valores inferiores a 6.11 ton $\mathrm{FV} / \mathrm{ha}$, por lo cual fueron diferentes entre sí $(\mathrm{P}<0.05)$. Así mismo la tendencia con la producción de materia seca (MS) (Figura 6) fue similar a la encontrada en FV, donde la dosis de cal E1 y E3 con potasio y nitrógeno mostraron los mayores contenidos con valores que oscilaron entre 2.0 y 2.16 toneladas de MS/ha, pero las dosis más altas de cal E4 con potasio y fósforo presentaron los menores contenidos de MS con valores entre 1.66 y 1.83 toneladas de $M S / h a(P<0.05)$. 


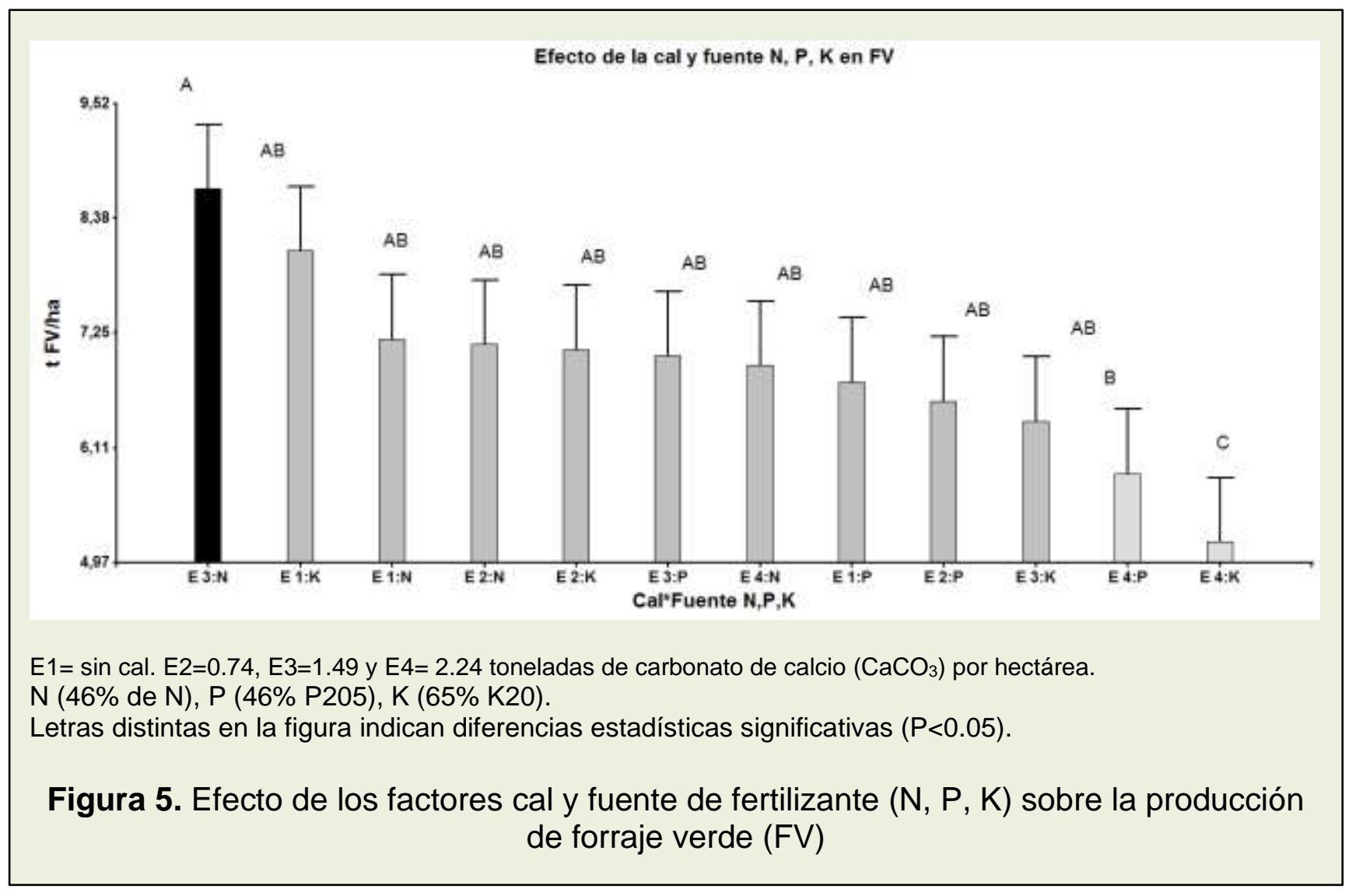

Los valores de aluminio y $\mathrm{pH}$ están relacionados, presentando una variación notable del Al entre las pasturas (Figura 1). De acuerdo al análisis de suelo (Tabla 1 ), en $B$. decumbens hubo mayor contenido de Al con las dosis más altas de cal E3 y E4, contrario a B. dictyoneura. Según Primavesi et al., (2005) con la aplicación de dosis más altas de calcio, éste puede ser lixiviado reduciéndose su efecto en la neutralización del aluminio; posiblemente se precipita en la forma iónica de nitrato de calcio, pudiendo aumentar en tres años la concentración de calcio intercambiable en 10 veces a una profundidad de un metro, principalmente en suelos con 25 a $30 \%$ de arcilla. Esta afirmación puede explicar el comportamiento obtenido en $B$. decumbens, en donde las precipitaciones superaron los $3000 \mathrm{~mm} / \mathrm{año}$. Una de las grandes preocupaciones relacionadas con el uso de altas cantidades de cal para neutralizar el aluminio en suelos ácidos dominados por arcillas $1: 1$ es el posible efecto que puede traer sobre la oxidación intensa de la materia orgánica en su proceso de mineralización, puede ocurrir 
formación de moléculas orgánicas de pequeño peso molecular y haber acomplejamiento del calcio, que así puede ser lixiviado en profundidad (Ziglio et al., 1995).

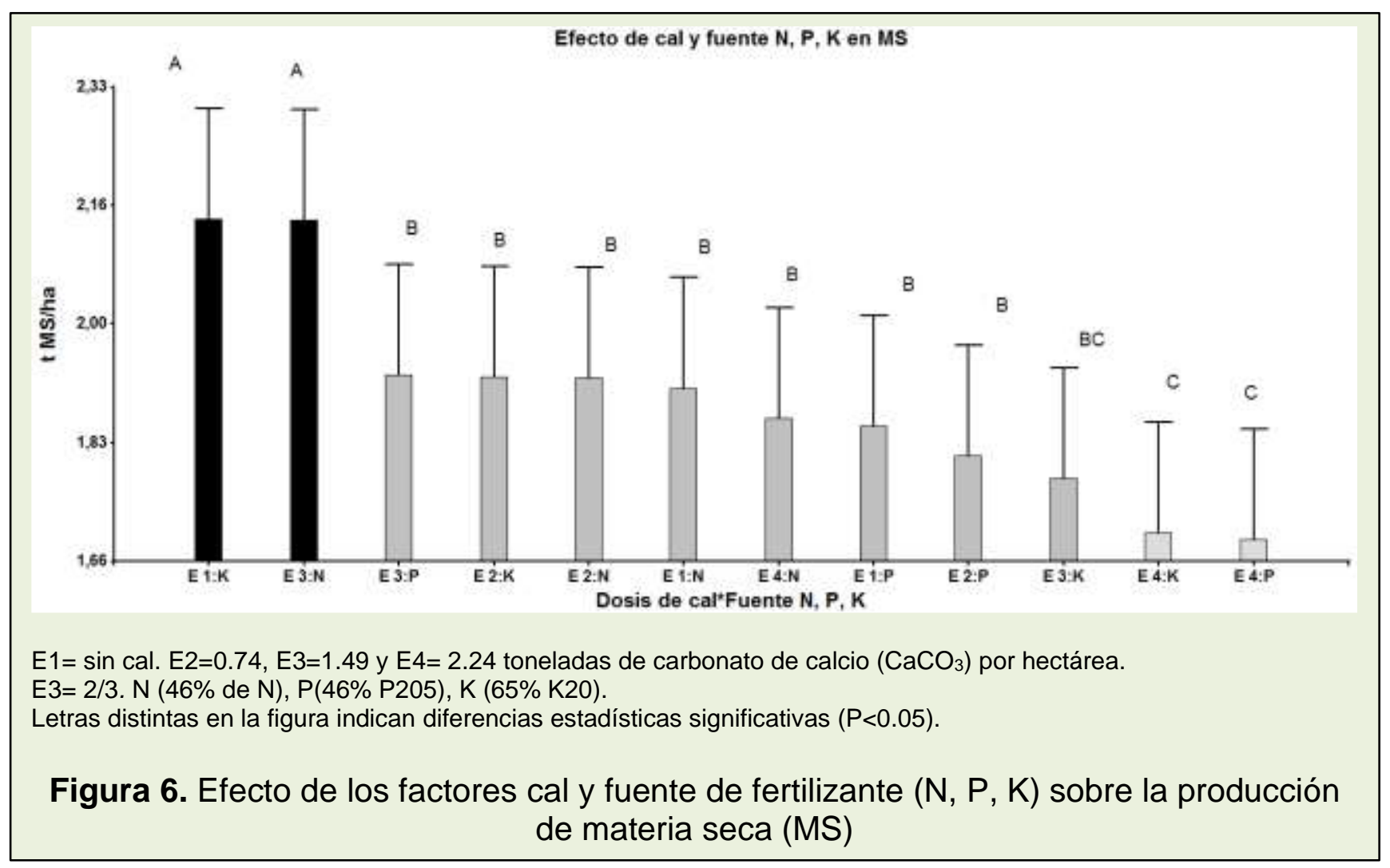

Por otra parte $B$. dictyoneura respondió mejor a la dosis de cal más baja E2 en la reducción de aluminio (Figura 1), dicho resultado podría ser interesante a la hora de recomendar el tipo de pastura y la dosis de cal más apropiada en la zona de estudio para la reducción de la fitotoxicidad de este elemento, aunque este no haya sido neutralizado en su totalidad. Según Paulino et al., (1994) los contenidos de aluminio son disminuidos con las dosis crecientes de cal, no ocurriendo neutralización total del aluminio intercambiable.

En suelos con $B$. dictyoneura se generaron mayores valores de $\mathrm{pH}$ y menores de aluminio intercambiable, al contrario de lo sucedido en la otra gramínea (Figura 2), lo cual fue corroborado por Paulino y Teixeira, (2010) debido a la tolerancia que tienen estas plantas a la acidez de los suelos y una relativa adaptación a las 
condiciones de baja fertilidad, más sin embargo este grado varía entre ellas, dependiendo de sus características genéticas. Grundy et al., (2001) encontraron que $B$. decumbens es tolerante a la toxicidad de aluminio, lo cual es ratificado por los resultados de este experimento. Se observó que $B$. dictyoneura presentó mayores contenidos de materia orgánica debido al mayor incremento en $\mathrm{pH}$ y disminución de los contenidos de aluminio, aunque siguen estando en un rango considerado como alto. Según Hartwig et al., (2007) los mecanismos fisiológicos de este tipo de pasturas pueden resultar en la activación de aniones de ácidos orgánicos como citrato que actúan como quelantes del aluminio y el secuestro de estos complejos por las vacuolas, disminuyendo su efecto nocivo en los suelos y favoreciendo un mayor desarrollo de las plantas. Por su parte el efecto de un mayor contenido de aluminio en los suelos pudo haber influido en menores contenidos de fósforo disponible en el caso de $B$. decumbens, porque el $\mathrm{Al}$ inhibe el crecimiento radicular de las plantas, reduciendo los cationes $\left(\mathrm{Ca}^{2+}, \mathrm{Mg}^{2+} \mathrm{y} \mathrm{K}^{+}\right)$y la disponibilidad de fósforo en los suelos y su consecuente absorción, siendo la principal limitante en los suelos en donde sembraron estas dos gramíneas.

La ausencia de fertilización con nitrógeno, fósforo y potasio en la fase de establecimiento de la pastura y la reposición de nutrientes extraídos por las pasturas tropicales, pueden ser los principales factores incidentes en la degradación de éstas (Anchão et al., 2003). A pesar de la baja disponibilidad de fósforo, el uso de fertilizantes fosfatados se complica debido al hecho de que éstos suelos presentan alta capacidad de fijación de fosfatos transformándolos en formas no disponibles para la planta como el fosfato de aluminio que es favorecido por la alta acidez intercambiable (Macedo, 2004; Castro y Gómez, 2010) por lo tanto, este factor incide posiblemente en un menor desarrollo radicular de las pasturas, lo que se tradujo en menores contenidos de materia orgánica. Las pasturas respondieron bien a las fertilizaciones con nitrógeno (Figura 4) lo que aumentó el contenido de materia orgánica del suelo, puesto que la principal deficiencia es dicho elemento, manifestándose con un bajo contenido de proteína en los forrajes. La eficiencia del uso de $\mathrm{N}$ disminuye con el aumento de sus dosis y tiende a incrementarse a través de los años, por el efecto del $\mathrm{N}$ residual y su 
liberación cuando esta inmovilizado (Primavesi et al., 2005; de Pinho et al., 2009). Según Rincón et al., (2008) los pastos adaptados a las condiciones de estos suelos ácidos han presentado buena respuesta a la aplicación de nitrógeno, fosforo, calcio, potasio, magnesio y azufre.

B. decumbens presentó mayor producción de FV y MS que B. dictyoneura (Figuras 5 y 6), como claramente fue demostrado por Ferreira et al., (2007) quienes confirman que la producción de forraje de especie Brachiaria es muy variable. La diferencia entre las producciones de biomasa fresca y seca en ambas especies de pastos, estuvo relacionada por las características propias de las pasturas y la tolerancia de $B$. decumbens a las condiciones de acidez, en adición a que no fue influenciado por los factores limitantes como el alto nivel de Al, bajos pH y contenido de MO y P (Reyes et al., 2009).

Rosas et al., (2017) demostraron que las barreras apoplásticas podrían determinar la resistencia al aluminio entre especies de Brachiaria. La eficiencia en la absorción de fósforo se refiere a la habilidad de las gramíneas para tomar gran cantidad del elemento por unidad de longitud de raíz cuando se siembran en condiciones de baja disponibilidad de dicho elemento (Carvalho et al., 2002). B. decumbens pudo presentar una mayor producción que la otra gramínea bajo las mismas condiciones ambientales y de cultivo. Referente a esto Rao et al., (1995) reportaron mayor eficiencia de uso de fósforo en $B$. decumbens que en otros cultivares de la misma especie, mientras que Correa y Haag, (1993) encontraron mayor eficiencia en $B$. decumbens con $B$. brizantha y $P$. maximun y en términos generales su mayor eficiencia de uso permite que la especie persista en suelos con bajo contenido de fósforo. De acuerdo con Navajas, (2011) este pasto muestra una gran capacidad de absorción de fosforo, que es un mineral esencial en el desarrollo del sistema radicular y su aporte se ve reflejado en la producción de biomasa (Rincón et al., 2008), estos mismos sustentan que la producción de materia seca de $B$. decumbens se estabiliza en 1.89 toneladas por hectárea, con un período de descanso de 26 días, y evaluado a los 45 días supera las 2 ton/ha, coincidiendo con los resultados de este trabajo. 


\section{CONCLUSIONES}

Brachiaria decumbens mostró mejores características de producción de biomasa, lo cual es de especial interés para el sector ganadero, a pesar de comprobar que la alta acidez y aluminio intercambiable son factores limitantes porque determinan bajos contenidos de materia orgánica y fósforo.

La dosis alta de cal y urea mejoró la eficiencia del fósforo disponible en el suelo de las pasturas, lo cual generó mayor producción de biomasa de la pradera bajo condiciones de suelos ácidos.

Potencializar los factores de producción de $B$. dictyoneura en la zona sería importante para mejorar su producción, puesto que con esta especie se incrementa la materia orgánica, el fósforo disponible, al tiempo que se reduce el aluminio intercambiable y el $\mathrm{pH}$. Además, cuando se fertiliza con nitrógeno se mejoran en mayor medida que con el $B$. decumbens, los contenidos de $\mathrm{MO}$ y $\mathrm{pH}$

Con todas las dosis de encalado evaluadas se redujo el aluminio intercambiable del suelo. Aunque no se mostró por sí solo el efecto de la cal en las propiedades químicas del suelo y la planta, si se observó que en combinación con fertilizantes de nitrógeno, fósforo y potasio, la incorporación de nutrientes y cal evitó el deterioro de las pasturas.

\section{REFERENCIAS BIBLIOGRÁFICAS}

1. Anchão P.P., Boaretto A.E., Ocheuze P.C., Salles W., Corsi M. Liming and fertilization to restore degraded Brachiaria decumbens pastures grown on an entisol. Scientia Agricola. 60 (1): 125-131. 2003.

2. Arias S.C., Pérez J.C., Rueda O.M. Lixiviación de nitratos en dos suelos al alterar sus propiedades físicas. Revista Escuela de Ingeniería de Antioquia. (2): 35-40. 2004.

3. Auclerc A., Nahmani J., Aran D., Baldy V., Callot H., Gers C., Iorio E., Lapied E., Lassauce A., Pasquet A. Changes in soil macroinvertebrate communities following liming of acidified forested catchments in the Vosges Mountains (North-eastern France). Ecological engineering. 42 (2012): 260-269. 2012.

4. Carvalho V., Netto S., Vasconcellos C.A., Gilson E., de Moura C. Cinética de absorção de fósforo e crescimento do sistema radicular de genótipos de milho contrastantes para eficiência a fósforo. Revista Brasileira de Milho e Sorgo. 1 (1): 8592. 2002.

5. Castro H., Gómez M. Fertilidad de suelos y fertilizantes. Sociedad Colombiana de la Ciencia del Suelo, Bogotá, Colombia. 27-303 p. 2010. 
6. Castro H., Munevar O. Mejoramiento químico de suelos ácidos mediante el uso combinado de materiales encalantes. Revista UDCA Actualidad \& Divulgación Científica. 16 (2): 409-416. 2013.

7. Civeira G., Rodríguez M.B. Nitrógeno residual y lixiviado del fertilizante en el sistema suelo-planta-zeolitas. Ciencia del suelo. 29 (2): 285-294. 2011.

8. Cochrane T., Salinas J., Sanchez P. An equation for liming acid mineral soils to compensate crop aluminium tolerance. Tropical Agriculture. 57 (2): 133-140. 1980.

9. CORPOICA, Corporación Colombiana de Investigación Agropecuaria. Fundamentos biofísicos y socioeconómicos para la formulación de propuestas productivas para la Orinoquia alta colombiana. Convenio MADR-CORPOICA, Villavicencio, Colombia. 206 p. 2007.

10. Correa L.d.A., Haag H. Níveis criticos de fósforo para o estabelecimento de gramineas forrageiras em Latossolo vermelho-amarelo, Alico: I: Ensaio em casa de vegetação. Embrapa Pecuária Sudeste-Artigo em periódico indexado (ALICE). 50 (1): 99-108. 1993.

11. de Pinho K.A., Pereira I., Faquin V., Pereira G., da Costa E. Produção de massa seca e nutrição nitrogenada de cultivares de Brachiaria brizantha (A. Rich) Stapf sob doses de nitrogênio Dry mass production and nitrogen nutritional value of Brachiaria brizantha (A. Rich) Stapf cultivars under nitrogen doses. Ciência e Agrotecnologia. 33 (6): 1578-1585. 2009.

12. Di Renzo J.A., Casanoves F., Balzarini M.G., Gonzalez L., Tablada M., Robledo C.W. InfoStat versión 2017. Grupo InfoStat, FCA. Universidad Nacional de Córdoba, Córdoba, Argentina. 2017. Recuperado 05 Jun 2017. Disponible En: http://www.infostat.com.ar

13. Ferreira A., Vieira A.J., Pinto G., Ferreira F., Silva R., Mattos C. Influência do nitrogênio e do fósforo na produção do capim-braquiária. Revista Brasileira de Zootecnia. 36 (5): 1240-1246. 2007.

14. Grundy S., Jones D., Godbold D. Organic acid root-tip tissue-concentration in Brachiaria decumbens and Brachiaria ruziziensis. Developments in Plant and Soil Sciences. Springer, p 506-507. 2001.

15. Hartwig I., Costa A., Irajá F., Bertan I., Gonzalez J.A., Mallmann D.A., Valério I.P., Maia L.C., Robe D.A., Sacramento C. Mecanismos associados à tolerância ao alumínio em plantas. Semina: Ciências Agrárias, Londrina. 28 (2): 219-228. 2007.

16. IGAC, Instituto Geográfico Agustín Codazzi. Métodos analíticos del laboratorio de suelos. Instituto Geográfico Agustín Codazzi, Bogotá, Colombia. 2003.

17. Jiménez O., Granados L., Oliva J., Quiroz J., Barrón M. Calidad nutritiva de Brachiaria humidicola con fertilización orgánica e inorgánica en suelos ácidos. Archivos de Zootecnia. 59 (228): 561-570. 2010.

18. Li W., Johnson C.E. Relationships among $\mathrm{pH}$, aluminum solubility and aluminum complexation with organic matter in acid forest soils of the Northeastern United States. Geoderma. 271 (1): 234-242. 2016.

19. Macedo M. Adubação fosfatada em pastagens cultivadas com ênfase na região do Cerrado. Associação Brasileira para a Pesquisa da Potassa e do Fosfato, Piracicaba, Brasil, p 359-396. 2004.

20. Navajas V. Efecto de la fertilización sobre la producción de biomasa y la absorción de nutrientes en Brachiaria decumbens y Brachiaria híbrido Mulato, Magister en Ciencias Agrarias. Facultad de Agronomía, Escuela de Posgrados, Universidad Nacional de Colombia, Bogotá, Colombia. 57 p. 2011.

21. Paulino V.T., Costa N., Cardelli L., Schammas E., Ferrari E. Resposta de Brachiaria brizantha cv. Marandu a calagem e a fertilização fosfatada em um solo ácido. Pasturas Tropicales (CIAT). 16 (2): 34-40. 1994. 
22. Paulino V.T., Teixeira E.M.d.L.C. Sustentabilidade de pastagens-manejo adequado como medida redutora da emissão de gases de efeito estufa. PUBVET, Publicações em Medicina Veterinária e Zootecnia. 4 (24, Ed 129): Art. 878, 18 p. 2010.

23. Posada S., Angulo J., Restrepo L. Validación de métodos de secado para la determinación de materia seca en especies forrajeras. Livestock Research for Rural Development. 19 (3): Art. 42.2007.

24. Primavesi O., Primavesi A.C., de Almeida L., Armelin M.J. Calagem em pastagem degradada de capim-braquiária ("Brachiaria Decumbes") intensamente adubada com nitrogênio em latossolo vermelho amarelo distrófico. En: 42 ${ }^{\underline{a}}$ Reunião Anual da Sociedade Brasileira de Zootecnia, p 1-6. 2005.

25. Quintero M.A., Furtado A.-A., Schramm M., Pinto F., Viana M., Virupax C. Aluminum effects on growth, photosynthesis, and mineral nutrition of cacao genotypes. Journal of plant nutrition. 36 (8): 1161-1179. 2013.

26. Rao I.M., Ayarza M.A., García R. Adaptive attributes of tropical forage species to acid soils I. Differences in plant growth, nutrient acquisition and nutrient utilization among C4 grasses and C3 legumes. Journal of plant nutrition. 18 (10): 2135-2155. 1995.

27. Reyes A., Bolaños E., Hernández D., Aranda E., Izquierdo F. Producción de materia seca y concentración de proteína en 21 genotipos del pasto humidícola Brachiaria humidicola (Rendle) Schweick. Universidad y ciencia. 25 (3): 213-224. 2009.

28. Rincón Á., Baquero J., Hernando F. Manejo de la nutrición mineral en sistemas ganaderos de los Llanos Orientales de Colombia. CORPOICA (Corporación Colombiana de Investigación Agropecuaria, Villavicencio, Meta, Colombia. 164 p. 2012.

29. Rincón Á., Bueno G., Mauricio Á., Pardo O., Pérez O., Caidedo S. Establecimiento, manejo y utilización de recursos forrajeros en sistemas ganaderos de suelos ácidos CORPOICA (Corporación Colombiana de Investigación Agropecuaria), Villavicencio, Meta, Colombia. 251 p. 2010.

30. Rincón Á., Ligarreto G., Garay E. Producción de forraje en los pastos Brachiaria decumbens cv. Amargo y Brachiaria brizantha cv. Toledo, sometidos a tres frecuencias ya dos intensidades de defoliación en condiciones del Piedemonte llanero colombiano. Revista Facultad Nacional de Agronomía. 61 (1): 4336 - 4346. 2008.

31. Rodríguez I., Crespo G., Fraga S. Efecto de las excreciones del ganado vacuno en el rendimiento y composición mineral del pasto y en la composición química del suelo. San José de las Lajas, La Habana. Cuba. 2001.

32. Rosas G., Puentes Y.J., Menjivar J.C. Relación entre el pH y la disponibilidad de nutrientes para cacao en un entisol de la Amazonia colombiana. Corpoica Ciencia y Tecnología Agropecuaria. 18 (3): 529-541. 2017.

33. Salinas J.G. Fertilización de pastos en suelos ácidos de los trópicos. Programa de Pastos Tropicales. Centro Internacional de Agricultura Tropical (CIAT), Cali, Colombia. 215 p. 1989.

34. Sánchez A.P., Salinas J.G. Suelos ácidos: estrategias para su manejo con bajos insumos en América tropical. Bogotá, Colombia. 93 p. 2008.

35. Zapata R.D. Química de la acidez del suelo. Ed Cargraphics, Cali, Colombia. 208 p. 2004.

36. Ziglio C.M., Miyazawa M., Pavan M.A. Mecanismo de deslocamento de cálcio no solo. En: Congresso brasileiro de ciência do solo. Viçosa, Brasil. 1995. Recuperado 05 Junio 2017.2 Disponible En: https://www.bdpa.cnptia.embrapa.br/consulta/busca?b=ad\&id=364333\&biblioteca=vaz io\&busca=autoria:\%22MIYAZAWA,\%20M:\%20PAVAN,\%20M.A.\%22\&qFacets=autori a:\%22MIYAZAWA,\%20M:\%20PAVAN,\%20M.A.\%22\&sort=\&paginacao=t\&paginaAtua $\underline{\underline{I}=1}$ 